\title{
Dynamical XPS measurements for probing photoinduced voltage changes
}

\author{
Hikmet Sezen, Sefik Suzer * \\ Department of Chemistry, Bilkent University, 06800 Ankara, Turkey
}

\section{A R T I C L E I N F O}

Article history:

Received 8 June 2010

Accepted 1 August 2010

Available online 6 August 2010

\section{Keywords:}

Dynamical XPS

Photovoltage

Photoconductivity

Charging

\begin{abstract}
A B S T R A C T
Photoillumination with $405 \mathrm{~nm}$ laser causes shifts in XPS peaks of n-Si(100), and CdS. To distinguish between surface photovoltage (SPV), and charging, dynamical measurements are performed, while sample is subjected to square wave pulses of $\pm 10.00 \mathrm{~V}$ amplitude, and $10^{-3}-10^{5} \mathrm{~Hz}$ frequency. For $\mathrm{n}-\mathrm{Si}$, Si2p peaks are twinned at +10.00 and -10.00 , yielding always $20.00 \mathrm{eV}$ difference. Photoillumination shifts the twinned peaks to higher energies, but the difference is always $20.00 \mathrm{eV}$. However, for CdS, the measured binding difference of $\mathrm{Cd} 3 \mathrm{~d}$ peaks exhibits strong frequency dependence due to charging, which indicates that both fast SPV and slow charging effects are operative.
\end{abstract}

(C) 2010 Elsevier B.V. All rights reserved.
Better understanding of the nature and control of the chemical and/ or physical changes associated with photoinduced processes is vital for numerous chemical and biochemical processes as well as their applications like in photovoltaics, sensors, etc. One consequence of the photoinduced changes is the development of an electrical voltage (photovoltage, or surface photovoltage) on different regions of the sample under investigation, which has been extensively investigated using electrical, electrochemical, spectroscopic, and scanning probe microscopic techniques, etc. [1-3]. Use of photoelectron spectroscopy for probing the so-called surface photovoltage (SPV), and/or other photoinduced voltage changes, is very appealing, since the observed features are sensitive to small voltage developments down to the orders of $10 \mathrm{meV}$, and chemically specific information can be harvested in addition [4-18]. In XPS analysis of surface structures, the voltage development manifests itself as shifts in the position of the core level peaks, since the detected photoelectrons' kinetic energy is modified by the developed voltage $[4,6,7,11-18]$. However, extreme care has to be exercised in sorting out the measured binding energy shifts, since charging can also cause shifts in the peak positions [10,19].

The SPV process for various semiconductors, has been investigated, in a very fast time-resolved mode (pico to microseconds), using mostly synchrotron radiation, in combination with various kinds of laser sources, utilizing pump-probe techniques [20-26]. We have recently developed a technique which probes the dynamical nature of the charging shifts by measuring the positions of the peaks when the sample is under the influence of a square wave pulse with varying frequencies in the range of $10^{-3}$ to $10^{5} \mathrm{~Hz}$ [27-32]. In this short contribution, we extend the application of our method to probing and

\footnotetext{
* Corresponding author. Tel.: +90 312 2901476; fax: +90 3122664068. E-mail address: suzer@fen.bilkent.edu.tr (S. Suzer).
}

differentiating between XPS shifts stemming from surface photovoltage and charging.

A clean Au metal, HF cleaned n-Si(100) wafer with a resistivity of 10 $20 \Omega \mathrm{cm}$, and a CdS film deposited on a $\mathrm{Si}(100)$ substrate are investigated. A Thermo Fisher K-Alpha electron spectrometer with monochromatic AlK $\alpha$ X-rays is used for XPS analysis, which is modified for imposing external voltage stress to the sample during data acquisition. Resolution of the spectrometer is better than $0.50 \mathrm{eV}$ as measured in the Si2p spin-orbit doublet with the $0.61 \mathrm{eV}$ spin-orbit splitting, and we estimate our precision to be better than $0.01 \mathrm{eV}$ for the measured binding energy differences. For probing the charging/ discharging properties, the samples are subjected to square wave pulses (SQW) of $\pm 10.00 \mathrm{~V}$ amplitude with varying frequencies in the range of $10^{-3}$ to $10^{5} \mathrm{~Hz}$, using a Stanford Research System DS340 pulse generator. For photoillumination, two $100 \mathrm{~mW}$ lasers at 405, and $532 \mathrm{~nm}$ (CrystaLaser) are employed in the C.W. mode, and their power densities are controlled by inserting various neutral density filters (NDF).

Shining light of appropriate frequency and intensity in a timedependent as well as in the C.W. mode, causes shifts in the positions of XPS peaks, due to a host of photochemical/photophysical processes, one of which is the fast and so-called surface photovoltage (SPV) effect. The SPV has long been investigated towards basic understanding of the underlying parameters involved for numerous semiconducting and dielectric materials [4-18,20-26]. As shown in Fig. 1, upon photoillumination with the $405 \mathrm{~nm}$ laser, the Si2p peaks of the $\mathrm{n}-\mathrm{Si}$ exhibit $+0.11 \mathrm{eV}$ shift, whereas the Au4f peaks (not shown) do not exhibit any measurable shift. As a result of pinning the Fermi level near the conduction band, there is already a $+0.12 \mathrm{eV}$ difference between the Si2p binding energy of our $\mathrm{n}-\mathrm{Si}$ (100) sample and that of an undoped Si sample, before any illumination, which is further affected by surface band-bending [20-26]. A similar shift is also observed using the $532 \mathrm{~nm}$ source. The overall effect of the photoillumination in semiconductors by light having energy larger 


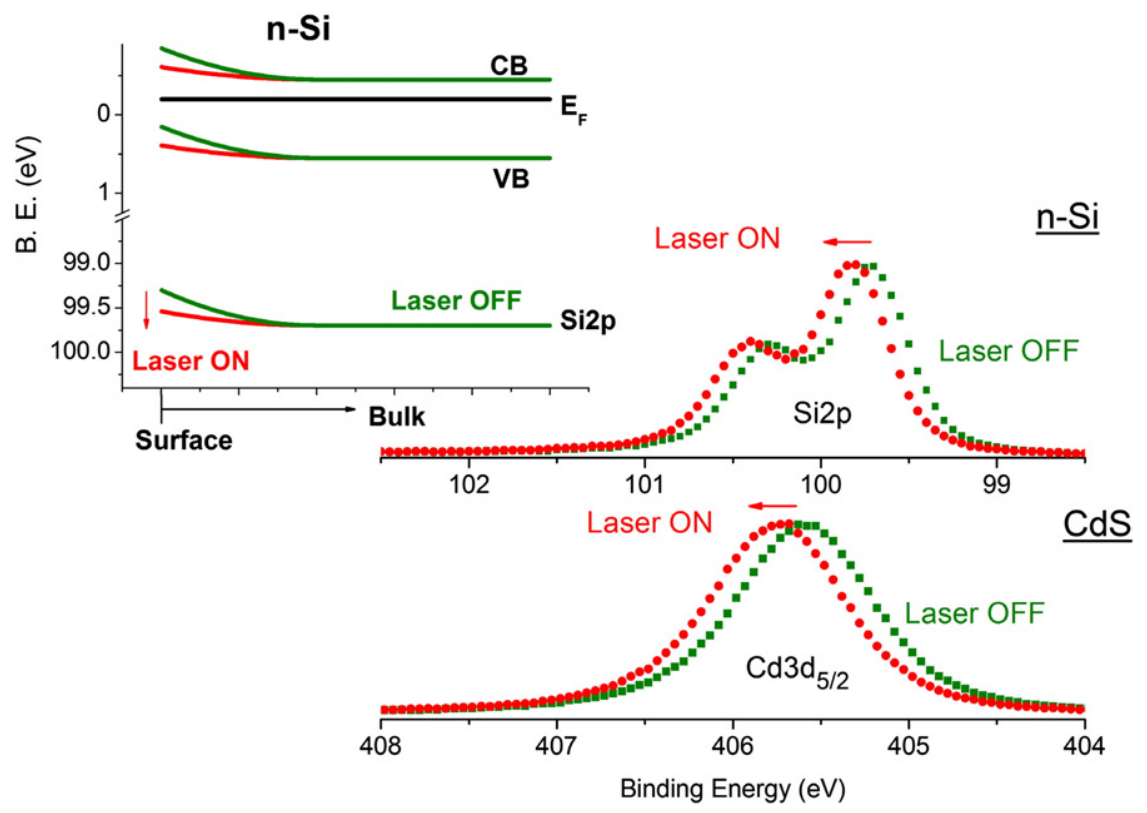

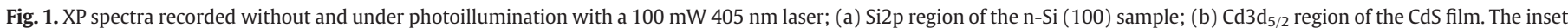
depicts the schematic band-bending diagrams of a n-Si sample without and under photoillumination.

that its band-gap (1.1 eV for $\mathrm{Si}$ ), is best described as decreasing the band-bending by creation of additional electron-hole pairs to result in further increasing of the measured binding energy of the Si2p for the case of $n-S i ́$, as depicted in the inset of the figure by the schematic band-diagram, consistent with our measurements [8,9,33].

CdS is a wide band-gap ( $2.4 \mathrm{eV})$ compound semiconductor which is intrinsically n-doped due to presence of ample S defects [34]. Hence, we expect and observe a similar photo behavior upon illumination with the $405 \mathrm{~nm}$ laser source, since it has an energy exceeding its band-gap. However, we also expect the film to exhibit charging shifts due to its significant resistivity [29]. Then, an important question arises as to how to differentiate between these two processes, which is the main objective of the present communication, where we will present a certain type of dynamical XPS measurements that can distinguish between the two.
The dynamical XPS measurements is best illustrated by using an artificial dielectric system consisting of a good conductor (Au metal) and connecting it through an external series resistor ( $R$ ), and a parallel capacitor (C) as shown in Fig. 2, which displays the Au4f spin-orbit doublet recorded; (a) when the sample is grounded, and (b) while subjected to a square wave excitation with $\pm 10.00 \mathrm{~V}$ amplitude and at frequencies of $100 \mathrm{~Hz}$ and $0.001 \mathrm{~Hz}$. This procedure simply twins the peaks at correspondingly $+10.00 \mathrm{eV}$ and $-10.00 \mathrm{eV}$ positions, with a measured binding energy difference of exactly $20.00 \mathrm{eV}$, since the sample is found to be caught $50 \%$ of its time at one position or the other, at all measured frequencies. An asymmetry, corresponding to a measured binding energy difference of less than $20.00 \mathrm{eV}$, can only be encountered after inclusion of a series resistor $(R=200 \mathrm{k} \Omega)$ externally, since different flooding electron currents are effective, when the sample is biased at $+10.00 \mathrm{~V}$ and $-10.00 \mathrm{~V}$. The net result is that the

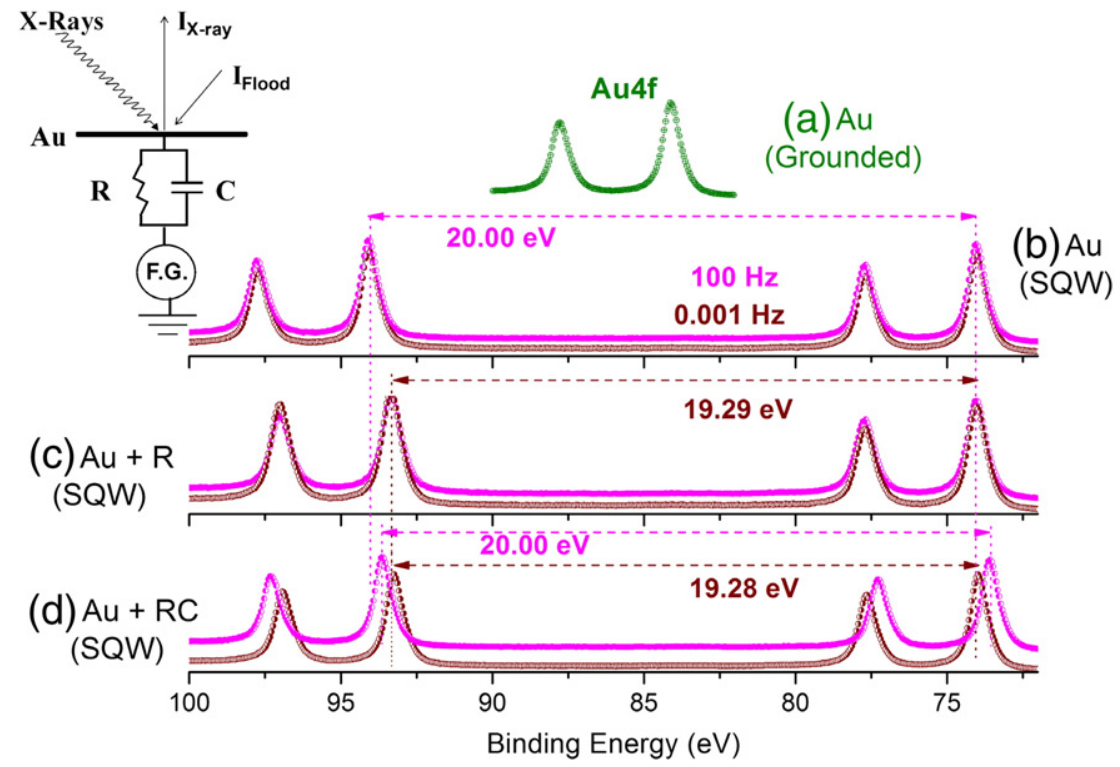

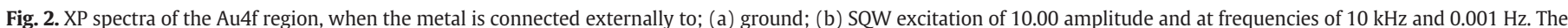

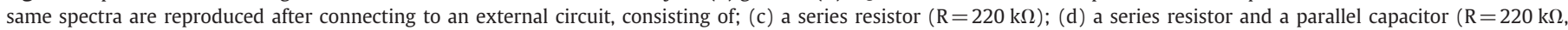

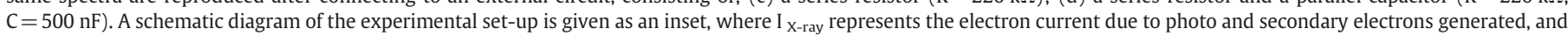
$\mathrm{I}_{\text {Flood }}$ is the current due to the flood-gun used. 
(a) Grounded

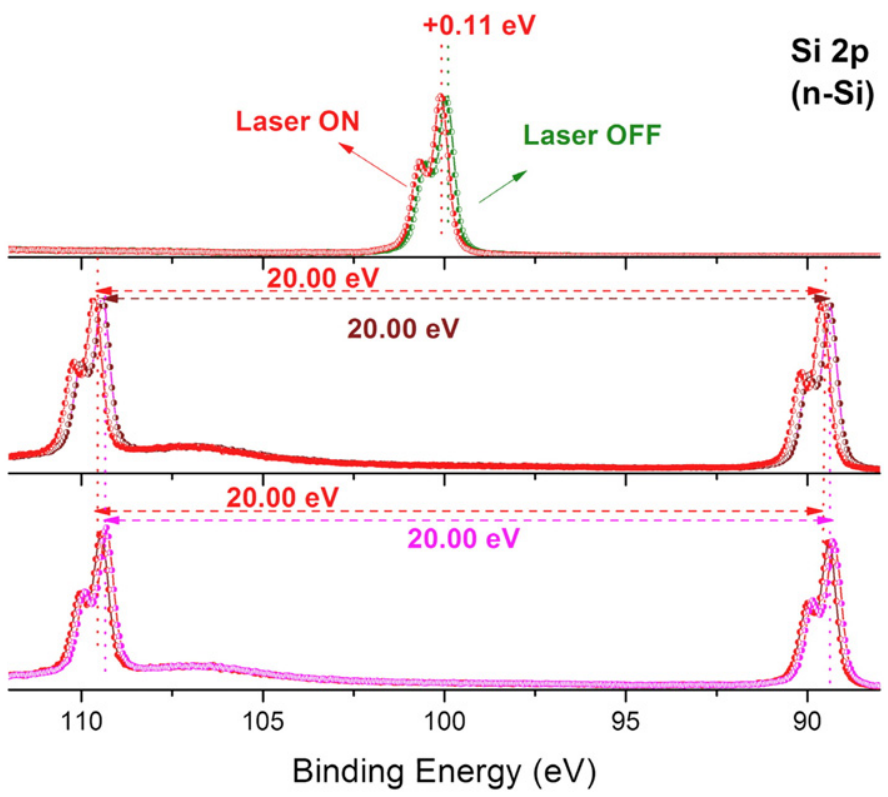

Fig. 3. (a) The same spectra of the $\mathrm{n}-\mathrm{Si}$ as in Fig. 1(a), and after subjecting to $0.001 \mathrm{~Hz}$ (b), and $10 \mathrm{kHz}$ (c) of the SQW excitation, without and under photoillumination.

measured binding energy difference is reduced from $20.00 \mathrm{eV}$ depending on the value of the resistor and/or the flooding electron current, but there is no frequency dependence at all, as shown in Fig. 2(c). The frequency (or time) dependence can be encountered only after introduction of an additional external parallel capacitor $(\mathrm{C}=500 \mathrm{nF}$ ) as shown in Fig. 2(d), where at low frequencies the system has ample time to charge and discharge and the binding energy difference becomes equivalent to the case of the resistor-only, and less than $20.00 \mathrm{eV}$ difference is measured. At sufficiently high frequencies, the capacitor is by-passed and the system does not have time to charge or discharge, and the $20.00 \mathrm{eV}$ difference is resumed. Note that, in the latter case, the energy scale is slightly off-set, due to the average current operative, which corresponds to $\mathbf{I}_{\mathbf{a v g}} \cdot \mathbf{R}$ voltage drop [31,32]. As a summary of these measurements, we can now propose that, under a SQW excitation of $10.00 \mathrm{~V}$ amplitude, the measured binding energy difference of less than $20.00 \mathrm{eV}$ reflects the presence of a measurable resistive path, and the change of this difference with frequency of the excitation reflects the presence of a measurable capacitative path, respectively. These are important outcome of our measurements, which, in the present communication, will be used as practical guidelines for characterizing certain photoinduced, or any other type of voltage changes we record for different samples, using dynamical XPS measurements.

In Fig. 3, we depict the Si2p peaks of a clean n-Si with and without photoillumination, recorded; (a) when the sample is grounded, and (b) $0.001 \mathrm{~Hz}$, and (c) $10 \mathrm{kHz} \mathrm{SQW}$ excitations. The photoillumination introduces SPV, which simply shifts the peaks to higher binding energy positions, but does not affect the measured binding energy difference at all for the measured frequencies from $10^{-3}$ to all the way up to $10^{5} \mathrm{~Hz}$. However, the situation for the CdS film is different, where both the binding energy difference of less than $20.00 \mathrm{eV}$, as well as a strong frequency dependence are measured, using both the 405 and $532 \mathrm{~nm}$ lasers, as shown in Fig. 4. Although, the energy of the $532 \mathrm{~nm}$ source is slightly below the band-gap of the bulk CdS, it seems
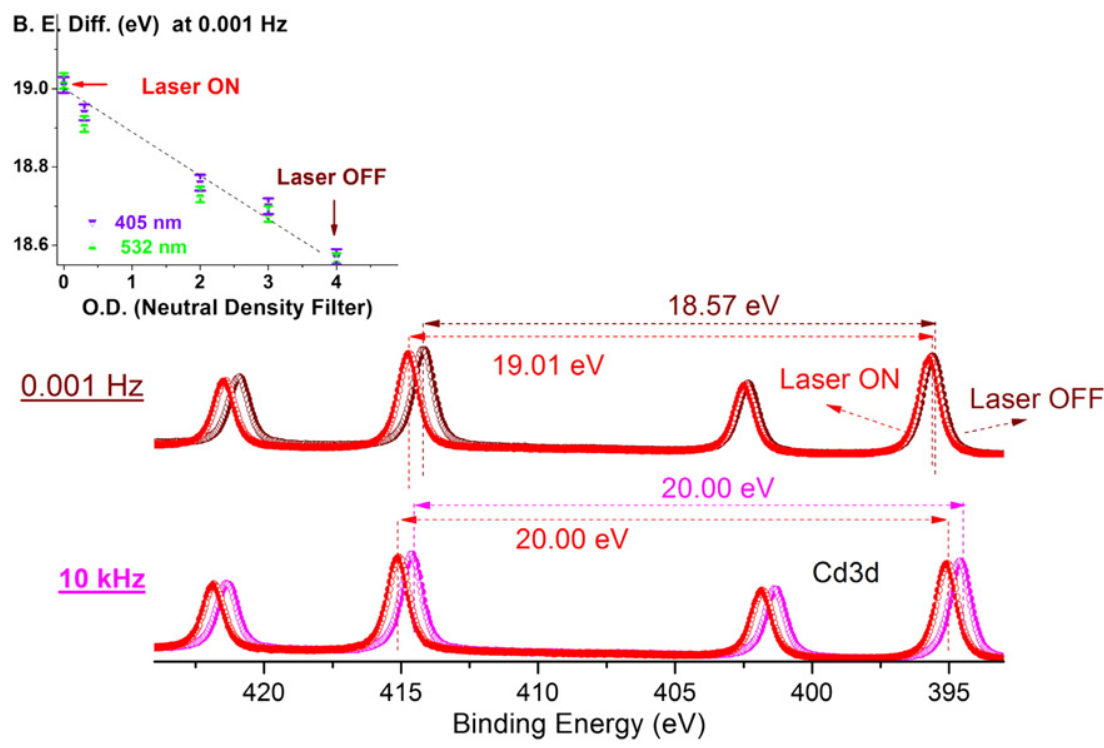

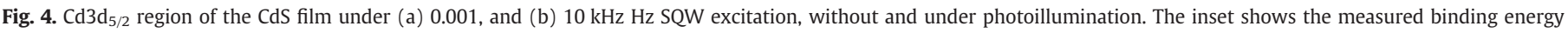

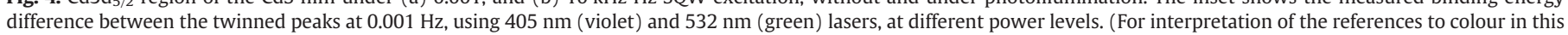
figure legend, the reader is referred to the web version of this article.) 
to be almost as effective as the $405 \mathrm{~nm}$ for our CdS film. Moreover, power dependence of the observed shifts is also very similar, as shown in the inset of Fig. 4.

The main reason why we can probe and differentiate between these two processes is related with their time scales. The SPV results from the electron-hole pair creation and has a life-time of pico to micro seconds, but under intense illumination, such as the case of our measurements, enough number of electron-hole pairs will survive to affect the measured voltage development(s). Since the electrons and the holes are mostly created in the conduction and the valence bands respectively, both their creation and recombination processes are fast, hence they cannot be probed by our relatively slow technique [2026]. However, in the CdS film, there are also other processes affecting the conductivity such as charging, which is relatively slow, hence can be probed by our slower but yet dynamical measurements [27-32]. The charging shifts are related with trapping and detrapping of the holes created following the very fast $\left(10^{-12} \mathrm{~s}\right)$ photoemission process, where after several cascading processes the holes are left as defect sites within the band-gap of the film. These processes have some relatively slow (in the orders of sub-seconds to minutes) components as was recently reported by Hirose by time-dependent XPS measurements $[19,35,36]$. Among many others, photoillumination by both the 532 and $405 \mathrm{~nm}$ lasers, seems to be contributing to both the fast SPV and the slow charging processes for the CdS film.

In short, we show by measurements, that by recoding the frequency dependent shifts of the XPS core peaks, it is possible to probe and distinguish between fast (mostly electronic) SPV, and slow (probably ionic) charging changes upon photoillumination of surface structures in a chemically sensitive fashion. Such measurements are general and have the potential of probing a host of related processes for a variety of dielectric and/or semiconducting interfaces and materials, and under various (optical, electrical, chemical, thermal, mechanical, etc.) excitations. The main emphasis of this short communication is the introduction of the methodology, since XPS is commonly used by various researchers, and further investigations including doping levels, the CdS film thickness, and/or preparation routes (thermal, electrochemical, laser-ablated, sol-gel, etc.) will be the subject of our further investigation(s).

\section{Acknowledgements}

We thank Prof. Dr. Atilla Aydinli of Bilkent Physics Department for providing the CdS sample, and for various fruitful discussions. This work was partially supported by TUBA (Turkish Academy of Sciences).

\section{References}

[1] L. Kronik, Y. Shapira, Surf. Sci. Rep. 37 (1999) 1.

[2] L. Kronik, Y. Shapira, Surf. Interface Anal. 31 (2001) 954

[3] D.K. Schroder, Meas. Sci. Technol. 12 (2001) R16.

[4] R. Buller, H. Cohen, E. Minkin, R. Popovitz-Biro, E. Lifshitz, M. Lahav, Adv. Funct. Mater. 12 (2002) 713.

[5] H. Cohen, J. Electron. Spectrosc. Relat. Phenom. 176 (2010) 24.

[6] H. Cohen, R. Maoz, J. Sagiv, Nano Lett. 6 (2006) 2462.

[7] H. Cohen, S.K. Sarkar, G. Hodes, J. Phys. Chem. B 110 (2006) 25508.

[8] J.E. Demuth, B.N.J. Persson, A.J. Schellsorokin, Phys. Rev. Lett. 51 (1983) 2214.

[9] J.E. Demuth, W.J. Thompson, N.J. Dinardo, R. Imbihl, Phys. Rev. Lett. 56 (1986) 1408.

10] N.J. Halas, J. Bokor, Phys. Rev. Lett. 62 (1989) 1679.

[11] N.R. Huck, R.S.C. Smart, S.M. Thurgate, Surf. Sci. 169 (1986) L245.

[12] W. Jaegermann, C. Pettenkofer, B.A. Parkinson, Phys. Rev. B 42 (1990) 7487.

[13] A. Schellenberger, R. Schlaf, C. Pettenkofer, W. Jaegermann, Phys. Rev. B 45 (1992) 3538.

[14] A. Samokhvalov, R.W. Gurney, M. Lahav, S. Cohen, H. Cohen, R. Naaman, J. Phys. Chem. B 107 (2003) 4245.

[15] S.K. Sarkar, G. Hodes, L. Kronik, H. Cohen, J. Phys. Chem. C 112 (2008) 6564.

[16] R. Schlaf, A. Klein, C. Pettenkofer, W. Jaegermann, Phys. Rev. B 48 (1993) 14242.

[17] J.H. Yang, Y. Zidon, Y. Shapira, J. Appl. Phys. 91 (2002) 703.

[18] Y. Zidon, J.H. Yang, Y. Shapira, Appl. Phys. Lett. 81 (2002) 436.

19] K. Hirose, H. Nohira, K. Azuma, T. Hattori, Prog. Surf. Sci. 82 (2007) 3.

[20] D. Brocker, T. Giessel, W. Widdra, Chem. Phys. 299 (2004) 247.

[21] J.P. Long, H.R. Sadeghi, J.C. Rife, M.N. Kabler, Phys. Rev. Lett. 64 (1990) 1158.

[22] M. Marsi, R. Belkhou, C. Grupp, G. Panaccione, A. Taleb-Ibrahimi, L. Nahon, D. Garzella, D. Nutarelli, E. Renault, R. Roux, M.E. Couprie, M. Billardon, Phys. Rev. B 61 (2000) R5070.

23] M. Marsi, M.E Couprie, L. Nahon, D. Garzella, R. Bakker, A. Delboulbe, D. Nutarelli, R. Roux, B. Visentin, C. Grupp, G. Indlekofer, G. Panaccione, A. TalebIbrahimi, M. Billardon, Nucl. Instrum. Methods Phys. Res., Sect. A 393 (1997) 548.

[24] M. Marsi, M.E. Couprie, L. Nahon, D. Garzella, T. Hara, R. Bakker, M. Billardon, A Delboulbe, G. Indlekofer, A. TalebIbrahimi, Appl. Phys. Lett. 70 (1997) 895.

[25] M. Marsi, L. Nahon, M.E. Couprie, D. Garzella, T. Hara, R. Bakker, M. Billardon, A Delboulbe, G. Indlekofer, A. Taleb-Ibrahimi, J. Electron. Spectrosc. Relat. Phenom. 94 (1998) 149

[26] W. Widdra, D. Brocker, T. Giessel, I.V. Hertel, W. Kruger, A. Liero, F. Noack, V. Petrov, D. Pop, P.M. Schmidt, R. Weber, I. Will, B. Winter, Surf. Sci. 543 (2003) 87.

[27] H. Sezen, G. Ertas, A. Dana, S. Suzer, Macromolecules 40 (2007) 4109.

[28] H. Sezen, G. Ertas, S. Suzer, J. Electron. Spectrosc. Relat. Phenom. 178-179 (2010) 373.

[29] H. Sezen, S. Suzer, Journal of Vacuum Science and Technology A 28 (2010) 639.

[30] S. Suzer, A. Dana, J. Phys. Chem. B 110 (2006) 19112

[31] S. Suzer, H. Sezen, A. Dana, Anal. Chem. 80 (2008) 3931.

[32] S. Suzer, H. Sezen, G. Ertas, A. Dana, J. Electron. Spectrosc. Relat. Phenom. 176 (2010) 52.

[33] F.J. Himpsel, G. Hollinger, R.A. Pollak, Phys. Rev. B 28 (1983) 7014.

[34] D.P. Amalnerkar, Mater. Chem. Phys. 60 (1999) 1.

[35] K. Hirose, J. Electron. Spectrosc. Relat. Phenom. 176 (2010) 46.

[36] T. Wang, T.E. Chang, L.P. Chiang, C.H. Wang, N.K. Zous, C. Huang, IEEE Trans. Electron Devices 45 (1998) 1511. 